\title{
Assessing the benefits and risks of tight glycemic control in critically ill children
}

This article was published in the following Dove Press journal:

Pediatric Health, Medicine and Therapeutics

25 August 2014

Number of times this article has been viewed

\section{Sarah B Kandil \\ E Vincent Faustino}

Department of Pediatrics, Division of Pediatric Critical Care, Yale University School of Medicine, New Haven, CT, USA
Correspondence: Sarah B Kandil;

E Vincent Faustino

Department of Pediatrics,

Division of Pediatric Critical Care,

Yale School of Medicine, 333 Cedar

Street, PO Box 208064, New Haven,

CT 06520-8064, USA

Tel + I 203785465 |

Fax + I 2037857194

Email sarah.kandil@yale.edu;

vince.faustino@yale.edu
Abstract: The benefit of intravenous insulin for tight glycemic control (TGC) in critically ill patients is debatable. Initial single center trials on TGC in critically ill adults admitted to the surgical intensive care unit were promising and showed a decrease in mortality with TGC. However, subsequent multicenter trials were unable to replicate these findings, with one trial even showing increased mortality with TGC. In critically ill children, efficacy and safety of TGC is poorly understood. High rates of insulin-induced hypoglycemia with TGC are concerning. Currently, there are no recommendations on the use of TGC for critically ill children. This review summarizes the current literature on TGC in children, focusing on its benefits and risks.

Keywords: hyperglycemia, hypoglycemia, intensive care unit, insulin, glucose

\section{Introduction}

Hyperglycemia is common in critically ill nondiabetic adults and children and is associated with increased morbidity and mortality. ${ }^{1-5}$ The use of intravenous insulin infusion to control blood glucose to near normal ranges, often referred to as tight glycemic control (TGC) or intensive insulin therapy, remains highly debated. Over a decade ago, a group from Leuven, Belgium demonstrated in a single center trial a one third decrease in mortality in critically ill adults admitted to the surgical intensive care unit (ICU) with hyperglycemia using TGC compared with similar patients whose blood glucose was not controlled. ${ }^{6}$ They also showed a similar decrease in mortality with TGC for critically ill adults admitted to the medical ICU for at least 3 days. ${ }^{7}$ However, a large multicenter trial, the Normoglycemia in Intensive Care Evaluation-Survival Using Glucose Algorithm Regulation (NICE-SUGAR), was unable to replicate the findings of the Leuven group. In fact, they demonstrated increased mortality with TGC (odds ratio [OR] 1.14; 95\% confidence interval [CI] 1.02-1.28, $P=0.02$ ) in critically ill adults admitted to the medical ICU. ${ }^{8}$ Meta-analyses of randomized controlled trials (RCT) of adults showed no benefits in mortality, incidence of blood stream infections, or the requirement for renal replacement therapy with TGC in critically ill patients admitted to medical-surgical ICUs. Furthermore, the rates of hypoglycemia were higher with adults randomized to TGC than in those randomized to the control group. Among patients on TGC, parenteral nutrition was associated with decreased risk of death compared to those not receiving parenteral nutrition. ${ }^{9,10}$ It has been postulated that the high glucose loads from parenteral nutrition may explain the differences noted between the Leuven trials, which used higher rates of parenteral nutrition, and the NICE-SUGAR trial, which mainly used enteral nutrition. ${ }^{9}$ 
Following the results from the Leuven trials, professional societies recommended treatment of hyperglycemia to maintain blood glucose close to normal levels (blood glucose $<110 \mathrm{mg} / \mathrm{dL}$ ) in critically ill adults, similar to goals advocated for outpatient settings. ${ }^{11}$ These recommendations were revised following contradictory findings from the NICE-SUGAR trials and the increased rates of hypoglycemia with TGC. To date, professional societies such as the American Diabetes Association and Society for Critical Care Medicine recommend controlling blood glucose in a more liberal range of $140-180 \mathrm{mg} / \mathrm{dL}$ and $100-150 \mathrm{mg} / \mathrm{dL}$, respectively. ${ }^{12,13}$

The literature regarding the risks and benefits of TGC in critically ill children is sparse. Pediatric intensivists are cautious with TGC given concerns for hypoglycemia, especially in the setting of the developing brain. ${ }^{14}$ In a recent survey of pediatric intensivist, only $6 \%$ of providers preferred a blood glucose target range of $80-110 \mathrm{mg} / \mathrm{dL}$ compared to $43 \%$ of providers who were surveyed several years earlier $(P<0.001) .{ }^{15}$ Currently, seven RCTs have evaluated the safety and efficacy of TGC in critically ill children, two of which are in premature infants (Table 1). This review will summarize the benefits and risks of TCG based on the RCTs on TGC in critically ill children.

\section{Pathophysiology}

Hyperglycemia during critical illness had always been thought of as the body's normal response to illness and often referred to as stress hyperglycemia. It is quite common in critically ill patients, with prevalence that can be as high as $75 \%$ depending on the blood glucose cutoff used to define hyperglycemia. ${ }^{2}$ Stress hyperglycemia is thought to ensure that vital tissues are provided with a constant supply of glucose during critical illness. Studies have shown that it results from increased gluconeogenesis in the liver and peripheral insulin resistance primarily in skeletal muscles. ${ }^{4}$ In adults, primary insulin resistance seems to be the key mechanism for hyperglycemia, while in children, beta-cell dysfunction is thought to play a more important role. ${ }^{5}$

Literature now suggests that stress hyperglycemia may not be as advantageous as previously thought. Stress hyperglycemia is associated with increased mortality and longer ICU stays in critically ill nondiabetic children. ${ }^{2}$ Prolonged hyperglycemia may lead to osmotic diuresis with subsequent hypovolemia, electrolyte abnormalities, and hyperosmolar nonketotic coma. Stress hyperglycemia itself can worsen catabolism within skeletal muscle and lead to increased rates of infection after surgery. It is also associated with depressed immune function, with inhibition of interleukin- 1 and diminished oxygen free radical production from neutrophils. ${ }^{16} \mathrm{The}$ causality of these associations has been investigated in the different RCTs on TGC in children.

\section{Efficacy of tight glycemic control in critically ill children}

Of the RCTs on TGC in critically ill children, the majority evaluated children recovering from cardiac surgery and used markers of inflammation or infection as outcome measures. This limits the extrapolation of their findings to other critically ill children. Vlasselaers et a ${ }^{17}$ were the first to address TGC in children in their single center trial. The majority of children enrolled had undergone cardiac surgery. The investigators found that children in the TGC group had lower levels of C-reactive protein (CRP) than those in the control group, suggesting that inflammation was less with TGC. TGC was also associated with shorter duration of ICU stay, less hemodynamic support, and lower levels of markers of cardiovascular dysfunction such as serum lactate and cardiac enzyme levels. They did not find any difference in markers of kidney or liver function. The trial had an alarmingly high rate of severe hypoglycemia (defined as $\leq 40 \mathrm{mg} / \mathrm{dL}$ ), which was nearly 25 times more common in the TGC group than in the control group. The hypoglycemic events, however, were not associated with seizures or death and were not associated with mortality after adjusting for ICU stay and baseline risk factors. ${ }^{17}$

Jeschke et al ${ }^{18}$ evaluated TGC in patients who suffered from severe burn injuries ( $>30 \%$ of their total body surface). The investigators found that the incidence of infection and sepsis in the TGC group were lower than those in the control group. Patients in the TGC group had lower levels of markers of inflammation, such as interleukin-6, CRP, complement C3, haptoglobin, and alpha 2-macroglobulin, than those in the control group. Normalization of hepatic and renal function was faster in the TGC than in the control group. Similar to the Vlasselaers trial, the incidence of severe hypoglycemia was higher in the TGC group.

Agus et $\mathrm{a}^{20}$ also focused on the effects TGC had on health care-associated infections in the Safe Pediatric Euglycemia after Cardiac Surgery (SPECS) study. This trial enrolled children recovering from surgical repair of their congenital heart defects. The investigators did not find any difference in the rates of infection (including pneumonia, bloodstream, urinary tract, or surgical site infections) between the TGC and control groups. This is in contrast to the prior trials, which suggested that TGC may mitigate the inflammatory 


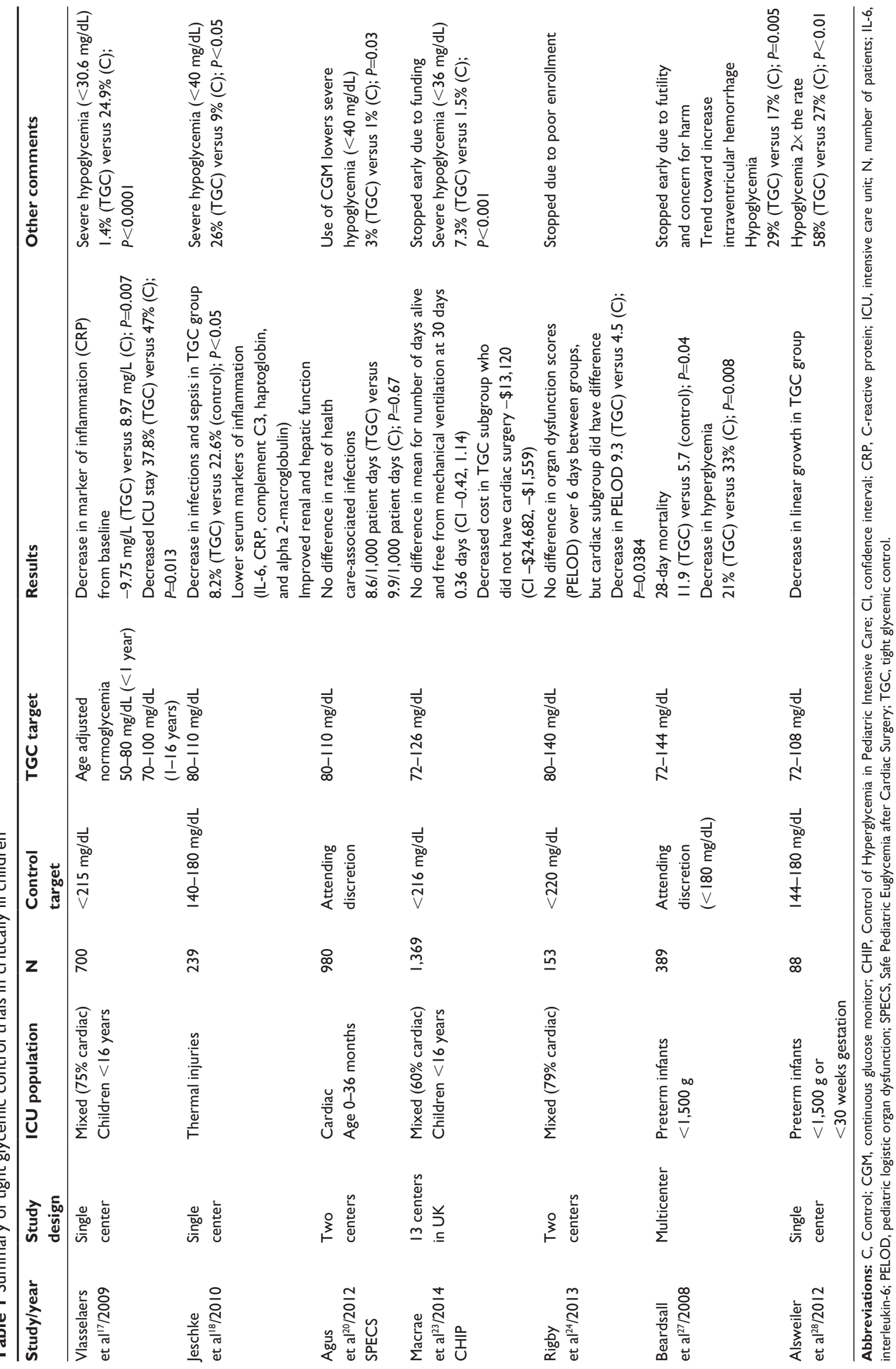


responses. In a post hoc analysis, however, the investigators showed that the effect of TGC may be related to the subjects' age. TGC was associated with higher rates of infections in children $\leq 60$ days old but with low rates of infections in older children. ${ }^{19}$ The investigators also did not find any differences in mortality, length of stay, or measures of organ dysfunction between the two groups. It is important to note that while this trial did not show any benefit for TGC in critically ill children, the investigators demonstrated that hypoglycemia with TGC can be reduced with the use of a continuous glucose monitor (CGM). With CGM, the risk of severe hypoglycemia with TGC was only $3 \%$ compared with $1 \%$ in the control group. ${ }^{20}$ These risks are comparable with the risk of spontaneous hypoglycemia in critically ill children. ${ }^{21,22}$ The SPECS study was the first trial to demonstrate that CGM can minimize the risk of hypoglycemia with TGC in critically ill children.

The largest trial on TGC in critically ill children to date is the Control of Hyperglycemia in Pediatric Intensive Care trial. ${ }^{23}$ In this multicenter trial, the investigators determined whether TGC reduced the number of days alive and free from mechanical ventilation within 30 days of trial entry. The investigators found no difference in this outcome between the TGC and control groups overall and in prespecified subgroups based on recent cardiac surgery, age group, and early versus late enrollment. As in prior studies, severe hypoglycemia was more common in the TGC than in the control group. ${ }^{23}$ An important contribution of this trial is cost analysis. The investigators compared the hospital costs between children in the TGC and control groups at 30 days and 12 months after randomization. The mean costs were similar between the two groups at 30 days although the costs seemed lower in the TGC group at 12 months.

Recently, Rigby et $\mathrm{al}^{24}$ conducted a small two-center RCT involving TGC in critically ill children. The trial was stopped early due to poor enrollment as they enrolled less than $20 \%$ of their estimated sample size. The investigators did not find any difference in change in organ dysfunction scores between TGC and control groups. Risks of hypoglycemia were not reported.

Because of the conflicting findings from the RCTs on TGC in critically ill children, Srinivasan and Agus ${ }^{25}$ performed a meta-analysis that included four of the five RCTs we have discussed in this review. The RCT by Rigby et al trial was not included because of limited available data. The meta-analysis, which included 3,288 subjects, found that TGC did not decrease 30-day mortality (OR 0.79; 95\% CI $0.55-1.15, P=0.22$ ) although the rates of acquired infection were lower with TGC (OR 0.76; CI 0.59-0.99, $P=0.04$ ). The risk of hypoglycemia was higher in subjects in the TGC than in the control group (OR 6.14; CI 2.74-13.78, $P<0.001){ }^{25}$

Hyperglycemia is also common in preterm neonates and is associated with adverse outcomes. ${ }^{26}$ Two multicenter RCTs on TGC in preterm neonates have been conducted. The trial by Beardsall et a ${ }^{27}$ showed increased mortality with TGC while the trial by Alsweiler et $\mathrm{al}^{28}$ showed decreased linear growth but increased head circumference and weight gain with TGC. Both trials had higher risks of hypoglycemia in neonates in the TGC than in the control group. The risk of hypoglycemia in the TGC group was significantly higher than in older children treated with TGC. ${ }^{27,28}$

\section{Improving the safety of tight glycemic control in critically ill children}

Insulin-induced hypoglycemia is thought to be the biggest risk associated with TCG. Many question if the risks of insulin-induced hypoglycemia carry the same risks as spontaneous hypoglycemia, which itself is common in critically ill patients. ${ }^{22}$ All except one RCT in critically ill children showed a high risk of hypoglycemia with TGC; a risk that is at least double those whose blood glucose was not controlled. ${ }^{17,18,23,29}$ In a survey by Preissig and Rigby ${ }^{30}$ of pediatric ICU centers across the United States, $60 \%$ of centers felt hypoglycemia was more harmful than hyperglycemia, and $70 \%$ listed fear of insulin-induced hypoglycemia as a barrier to starting TGC.

Although hypoglycemia can lead to seizures, coma, and eventually death, if unrecognized or untreated, we previously showed that hypoglycemia is likely a marker and not a cause of increased mortality in critically ill children. ${ }^{21,22}$ Vlasselaers et $\mathrm{al}^{17}$ showed that hypoglycemia was not associated with mortality when they adjusted for ICU stay, or other clinically relevant baseline risk factors such as age and weight. These findings are in contrast to those from adults. Post hoc analysis of the data from the NICE-SUGAR trial showed that those with moderate or severe hypoglycemia had increased risk of death. ${ }^{31}$ Neurocognitive outcomes of children with hypoglycemia 4 years after enrollment in the trial were not different from age-matched children from the trial's control group. Neurologic outcomes are also being evaluated for those enrolled in the SPECS trial. Results are eagerly awaited.

While the sequelae of hypoglycemia in critically ill children remain unclear, it is prudent to mitigate the risks of 
hypoglycemia. The use of CGM needs to be further explored. The SPECS trial was the first to report a reduction in the risk of hypoglycemia with the use of CGM in combination with a computerized algorithm. ${ }^{20}$ While the use of CGM may increase nursing workload due to high rate of false negative alarms, CGMs likely will improve overall safety of TGC.

\section{HALF-PINT trial}

Currently there is an additional ongoing multicenter RCT on TGC in critically ill children. This RCT, Heart and Lung Failure-Pediatric Insulin Titration (HALF-PINT), is enrolling critically ill children who did not undergo cardiac surgery. The RCT is using CGM in combination with a computerized algorithm to minimize hypoglycemia. The goal is to enroll 1,880 patients to evaluate the effects of TGC on 28-day survival adjusted for ICU length of stay (ClinicalTrials. gov; NCT01565941). The trial will also evaluate the effect of TGC on the long-term neurocognitive outcomes of critically ill children.

\section{Conclusion}

The efficacy of TGC in critically ill children remains uncertain. TGC does not seem to affect mortality although it may reduce the rates of acquired infections. Because most of the RCTs in critically ill children enrolled those recovering from cardiac surgery, it is unclear whether these results can be extrapolated to other groups of critically ill children. TGC is associated with increased risk of hypoglycemia. However, the use of CGM may reduce this risk. HALF-PINT is currently enrolling critically ill children who did not undergo cardiac surgery. The trial is using CGM to minimize the risk of hypoglycemia. We are expecting that this trial will clarify the efficacy and safety of TGC in this group of critically ill children.

\section{Acknowledgments}

This publication was made possible by Clinical and Translational Science Award grants numbers UL1 TR000142 and KL2 TR000140 from the National Center for Research Resources and the National Center for Advancing Translational Science, components of the National Institutes of Health (NIH), and NIH roadmap for Medical Research. Its contents are solely the responsibility of the authors and do not necessarily represent the official view of the NIH.

\section{Disclosure}

The authors report no conflicts of interest in this work.

\section{References}

1. Capes SE, Hunt D, Malmberg K, Gerstein HC. Stress hyperglycaemia and increased risk of death after myocardial infarction in patients with and without diabetes: a systematic overview. Lancet. 2000;355(9206): 773-778.

2. Faustino EV, Apkon M. Persistent hyperglycemia in critically ill children. J Pediatr. 2005;146(1):30-34.

3. Bhutia TD, Lodha R, Kabra SK. Abnormalities in glucose homeostasis in critically ill children. Pediatr Crit Care Med. 2013;14(1):e16-e25.

4. Van Cromphaut SJ. Hyperglycaemia as part of the stress response: the underlying mechanisms. Best Pract Res Clin Anaesthesiol. 2009;23(4): 375-386.

5. Preissig CM, Rigby MR. Hyperglycaemia results from beta-cell dysfunction in critically ill children with respiratory and cardiovascular failure: a prospective observational study. Crit Care. 2009;13(1): R27.

6. van den Berghe $\mathrm{G}$, Wouters $\mathrm{P}$, Weekers $\mathrm{F}$, et al. Intensive insulin therapy in critically ill patients. $N$ Engl J Med. 2001;345(19):1359-1367.

7. van den Berghe G, Wilmer A, Hermans G, et al. Intensive insulin therapy in the medical ICU. N Engl J Med. 2006;354(5):449-461.

8. Finfer S, Chittock DR, Su SY, et al; NICE-SUGAR Study Investigators. Intensive versus conventional glucose control in critically ill patients. N Engl J Med. 2009;360(13):1283-1297.

9. Marik PE, Preiser JC. Toward understanding tight glycemic control in the ICU: a systematic review and metaanalysis. Chest. 2010;137(3): 544-551.

10. Wiener RS, Wiener DC, Larson RJ. Benefits and risks of tight glucose control in critically ill adults: a meta-analysis. JAMA. 2008;300(8): 933-944.

11. Garber AJ, Moghissi ES, Bransome ED Jr, et al; American College of Endocrinology Task Force on Inpatient Diabetes Metabolic Control. American College of Endocrinology position statement on inpatient diabetes and metabolic control. Endocr Pract. 2004;10 Suppl 2:4-9.

12. Moghissi ES, Korytkowski MT, DiNardo M, et al; American Association of Clinical Endocrinologists; American Diabetes Association. American Association of Clinical Endocrinologists and American Diabetes Association consensus statement on inpatient glycemic control. Diabetes Care. 2009;32(6):1119-1131.

13. Jacobi J, Bircher N, Krinsley J, et al. Guidelines for the use of an insulin infusion for the management of hyperglycemia in critically ill patients. Crit Care Med. 2012;40(12):3251-3276.

14. Hirshberg E, Lacroix J, Sward K, Willson D, Morris AH. Blood glucose control in critically ill adults and children: a survey on stated practice. Chest. 2008;133(6):1328-1335.

15. Hirshberg EL, Sward KA, Faustino EV, et al. Clinical equipoise regarding glycemic control: a survey of pediatric intensivist perceptions. Pediatr Crit Care Med. 2013;14(2):123-129.

16. Mizock BA. Alterations in fuel metabolism in critical illness: hyperglycaemia. Best Pract Res Clin Endocrinol Metab. 2001;15(4): 533-551.

17. Vlasselaers D, Milants I, Desmet L, et al. Intensive insulin therapy for patients in paediatric intensive care: a prospective, randomised controlled study. Lancet. 2009;373(9663):547-556.

18. Jeschke MG, Kulp GA, Kraft R, et al. Intensive insulin therapy in severely burned pediatric patients: a prospective randomized trial. $\mathrm{Am}$ J Respir Crit Care Med. 2010;182(3):351-359.

19. Agus MS, Asaro LA, Steil GM, et al; SPECS Investigators. Tight glycemic control after pediatric cardiac surgery in high-risk patient populations: a secondary analysis of the safe pediatric euglycemia after cardiac surgery trial. Circulation. 2014;129(22):2297-2304.

20. Agus MS, Steil GM, Wypij D, et al; SPECS Study Investigators. Tight glycemic control versus standard care after pediatric cardiac surgery. N Engl J Med. 2012;367(13):1208-1219.

21. Faustino EV, Bogue CW. Relationship between hypoglycemia and mortality in critically ill children. Pediatr Crit Care Med. 2010;11(6): 690-698. 
22. Faustino EV, Hirshberg EL, Bogue CW. Hypoglycemia in critically ill children. J Diabetes Sci Technol. 2012;6(1):48-57.

23. Macrae D, Grieve R, Allen E, et al; CHiP Investigators. A randomized trial of hyperglycemic control in pediatric intensive care. NEngl J Med. 2014;370(2):107-118.

24. Rigby M, Maher K, Preissig C, et al. The PedIETrol Trial: a 2-center trial of glycemic control in pediatric critical illness. Critical Care Medicine. 2013;41(12):Abstract 993.

25. Srinivasan V, Agus MS. Tight glucose control in critically ill children - a systematic review and meta-analysis. Pediatr Diabetes. 2014;15(2):75-83.

26. Hays SP, Smith EO, Sunehag AL. Hyperglycemia is a risk factor for early death and morbidity in extremely low birth-weight infants. Pediatrics. 2006;118(5):1811-1818.

27. Beardsall K, Vanhaesebrouck S, Ogilvy-Stuart AL, et al. Early insulin therapy in very-low-birth-weight infants. N Engl J Med. 2008;359(18): $1873-1884$.
28. Alsweiler JM, Harding JE, Bloomfield FH. Tight glycemic control with insulin in hyperglycemic preterm babies: a randomized controlled trial. Pediatrics. 2012;129(4):639-647.

29. Agus MS, Steil GM, Wypij D, et al; SPECS Study Investigators. Tight glycemic control versus standard care after pediatric cardiac surgery. N Engl J Med. 2012;367(13):1208-1219.

30. Preissig CM, Rigby MR. A disparity between physician attitudes and practice regarding hyperglycemia in pediatric intensive care units in the United States: a survey on actual practice habits. Crit Care. 2010;14(1):R11.

31. Finfer S, Liu B, Chittock DR, et al; NICE-SUGAR Study Investigators. Hypoglycemia and risk of death in critically ill patients. N Engl J Med. 2012;367(12):1108-1118.

\section{Publish your work in this journal}

Pediatric Health, Medicine and Therapeutics is an international, peerreviewed, open access journal publishing original research, reports, editorials, reviews and commentaries. All aspects of health maintenance, preventative measures and disease treatment interventions are addressed within the journal. Practitioners from all disciplines are invited to submit their work as well as healthcare researchers and patient support groups. The manuscript management system is completely online and includes a very quick and fair peer-review system. Visit http://www.dovepress.com/ testimonials.php to read real quotes from published authors.

Submit your manuscript here: http://www.dovepress.com/pediatric-health-medicine-and-therapeutics-journal 